\title{
A Note on Extended Gaussian Quadrature Rules
}

\author{
By Giovanni Monegato*
}

\begin{abstract}
Extended Gaussian quadrature rules of the type first considered by Kronrod are examined. For a general nonnegative weight function, simple formulas for the computation of the weights are given, together with a condition for the positivity of the weights associated with the new nodes. Examples of nonexistence of these rules are exhibited for the weight functions $\left(1-x^{2}\right)^{\lambda-1 / 2}, e^{-x^{2}}$ and $e^{-x}$. Finally, two examples are given of quadrature rules which can be extended repeatedly.
\end{abstract}

1. Introduction. A quadrature rule of the type

$$
\int_{a}^{b} w(x) f(x) d x=\sum_{i=1}^{n} A_{i}^{(n)} f\left(\xi_{i}^{(n)}\right)+\sum_{j=1}^{n+1} B_{j}^{(n)} f\left(x_{j}^{(n)}\right)+R_{n}(f)
$$

where $\xi_{i}^{(n)}, i=1, \ldots, n$, are the zeros of the $n$ th-degree orthogonal polynomial $\pi_{n}(x)$ belonging to the nonnegative weight function $w(x)$, can always be made of polynomial degree $3 n+1$ by selecting as nodes $x_{j}^{(n)}, j=1,2, \ldots, n+1$, the zeros of the polynomial $p_{n+1}(x)$, of degree $n+1$, satisfying the orthogonality relation

$$
\int_{a}^{b} w(x) \pi_{n}(x) p_{n+1}(x) x^{k} d x=0, \quad k=0,1, \ldots, n .
$$

The polynomial $p_{n+1}(x)$ is unique up to a normalization factor and can be constructed, for example, as described by Patterson [4]. Unfortunately, the zeros of $p_{n+1}(x)$ are not necessarily real, let alone contained in $[a, b]$. We call $(1.1)$ an extended Gaussian quadrature rule, if the polynomial degree is $3 n+1$, and all nodes $x_{j}^{(n)}$ are real and contained in $[a, b]$.

The only known existence result relates to the weight function $w(x)=$ $\left(1-x^{2}\right)^{\lambda-1 / 2},-a=b=1,0 \leqslant \lambda \leqslant 2$, for which Szegö [9] proves that the zeros of $p_{n+1}(x)$ are all real, distinct, inside $[-1,1]$, and interlaced with the zeros $\xi_{i}^{(n)}$ of the ultraspherical polynomial $\pi_{n}(x)$.

Kronrod [3] considers the case $\lambda=1 / 2$ and computes nodes and weights for the corresponding rule (1.1) up to $n=40$. For the same weight function, Piessens [6] constructs an automatic integration routine using a rule of type (1.1) with $n=10$. Further accounts of Kronrod rules, including computer programs, can be found in [8], [2] .

Patterson [4] derives a sequence of quadrature formulas by successively iterating the process defined by (1.1) and (1.2). Starting with the 3-point Gauss-Legendre rule, he adds four new abscissas to obtain a 7-point rule, then eight new nodes to obtain a 15-point rule and continues the process until he reaches a 127-point rule. The procedure,

Received December 2, 1975.

AMS (MOS) subject classifications (1970). Primary 65D30; Secondary 33A65.

*Work performed while the author held a fellowship from the Consiglio Nazionale delle Ricerche, Italy.

Copyright $\odot$ 1976, American Mathernatical Society 
even carried one step further to include a 255 -point rule, is made the basis of an automatic numerical integration routine in [5].

Ramsky [7] constructs the polynomial $p_{n+1}(x)$ satisfying condition (1.2) for the Hermite weight function up to $n=10$ and notes that the zeros are all real only when $n=1,2,4$.

In all papers [3], [4] and [5], all weights are positive; however in [7], for $n=4$, two (symmetric) weights $A_{i}^{(n)}$ are negative.

We first study a rule of type (1.1) with polynomial degree at least $2 n$ and give simple formulas for the weights $A_{i}^{(n)}$ and $B_{j}^{(n)}$, together with a condition for the positivity of the weights $B_{j}^{(n)}$. We then construct the polynomial $p_{n+1}(x)$ in (1.2) for the weight functions $w(x)=\left(1-x^{2}\right)^{\lambda-1 / 2}$ on $[-1,1], \lambda=0(.5) 5,8, w(x)=e^{-x^{2}}$ on $[-\infty, \infty]$, and $w(x)=e^{-x}$ on $[0, \infty]$, in each case up to $n=20$, and give examples in which $p_{n+1}(x)$ has complex roots. We compute the extended Gaussian quadrature rules, whenever they exist, and give further examples of rules with negative weights $A_{i}^{(n)}$. Finally, we give two examples of quadrature rules which can be extended repeatedly.

2. The Weights $A_{i}^{(n)}$ and $B_{j}^{(n)}$. Let $k_{n}>0$ be the coefficient of $x^{n}$ in $\pi_{n}(x)$, and $h_{n}=\int_{a}^{b} w(x) \pi_{n}^{2}(x) d x$. Consider a rule of type (1.1) with real nodes $x_{j}^{(n)}, j=1,2$, $\ldots, n+1$, and polynomial degree at least $2 n$. Let $q_{n+1}(x)=\Pi_{j=1}^{n+1}\left(x-x_{j}^{(n)}\right)$ and define $Q_{2 n+1}(x)=\pi_{n}(x) q_{n+1}(x)$. We assume the two sets of nodes $\left\{\xi_{i}^{(n)}\right\}_{i=1}^{n}$ and $\left\{x_{j}^{(n)}\right\}_{j=1}^{n+1}$ both ordered decreasingly.

THEOREM 1. We have

$$
B_{j}^{(n)}=\frac{h_{n}}{k_{n} Q_{2 n+1}^{\prime}\left(x_{j}^{(n)}\right)}, \quad j=1,2, \ldots, n+1,
$$

and all $B_{j}^{(n)}>0$ if and only if the nodes $x_{j}^{(n)}$ and $\xi_{i}^{(n)}$ interlace.

Proof. Applying (1.1) to $f_{k}(x)=\pi_{n}(x) q_{n+1}(x) /\left(x-x_{k}^{(n)}\right), k=1,2, \ldots, n+1$, we obtain

$$
\int_{a}^{b} w(x) f_{k}(x) d x=B_{k}^{(n)} \pi_{n}\left(x_{k}^{(n)}\right) q_{n+1}^{\prime}\left(x_{k}^{(n)}\right)=B_{k}^{(n)} Q_{2 n+1}^{\prime}\left(x_{k}^{(n)}\right) .
$$

Since $q_{n+1}(x) /\left(x-x_{k}^{(n)}\right)=x^{n}+t_{n-1}(x)$, where $t_{n-1}(x)$ is a polynomial of degree at most $n-1$, we have, by the orthogonality of $\pi_{n}(x)$,

$$
\int_{a}^{b} w(x) f_{k}(x) d x=\int_{a}^{b} w(x) \pi_{n}(x) x^{n} d x=h_{n} / k_{n} .
$$

Since $h_{n} / k_{n}>0$, we see that $Q_{2 n+1}^{\prime}\left(x_{k}^{(n)}\right) \neq 0$, and (2.1) follows from (2.2) and (2.3). Note in particular that the nodes $x_{j}^{(n)}$ are simple and distinct from the $\xi_{i}^{(n)}$.

Assume now that the nodes $x_{j}^{(n)}$ and $\xi_{i}^{(n)}$ interlace, i.e., $x_{n+1}^{(n)}<\xi_{n}^{(n)}<x_{n}^{(n)}<$ $\cdots<\xi_{1}^{(n)}<x_{1}^{(n)}$. Since the polynomial $Q_{2 n+1}$ vanishes precisely at the nodes $x_{j}^{(n)}$ and $\xi_{i}^{(n)}$, and by normalization, $Q_{2 n+1}(x)>0$ for $x>x_{1}^{(n)}$, it is clear that the derivative $Q_{2 n+1}^{\prime}$ will be alternately positive and negative at the nodes $x_{1}^{(n)}, \xi_{1}^{(n)}, x_{2}^{(n)}, \xi_{2}^{(n)}$, $\ldots$, hence, in particular; $Q_{2 n+1}^{\prime}\left(x_{j}^{(n)}\right)>0, j=1,2, \ldots, n+1$. By (2.1), therefore, $B_{j}^{(n)}>0$. 
Vice versa, suppose the weights $B_{j}^{(n)}, j=1,2, \ldots, n+1$, are positive. Applying (1.1) to the function

$$
f_{i}(x)=\pi_{n}^{2}(x) /\left(\left(x-\xi_{i+1}^{(n)}\right)\left(x-\xi_{i}^{(n)}\right)\right), \quad i=1, \ldots, n-1,
$$

we obtain

$$
0=\int_{a}^{b} w(x) f_{i}(x) d x=\sum_{j=1}^{n+1} B_{j}^{(n)} f_{i}\left(x_{j}^{(n)}\right) .
$$

Since all the nodes $x_{j}^{(n)}$ are distinct from any $\xi_{i}^{(n)}$, the sum in (2.4) can be zero only if at least one of the numbers $f_{i}\left(x_{j}^{(n)}\right)$ is negative. It follows that at least one node $x_{j}^{(n)}$, say $x_{j_{i}}^{(n)}$, satisfies the inequality

$$
\xi_{i+1}^{(n)}<x_{j_{i}}^{(n)}<\xi_{i}^{(n)}, \quad i=1, \ldots, n-1 .
$$

The existence of nodes $x_{1}^{(n)}>\xi_{1}^{(n)}$ and $x_{n+1}^{(n)}<\xi_{n}^{(n)}$ follows similarly by considering $f_{0}(x)=\pi_{n}^{2}(x) /\left(\xi_{1}^{(n)}-x\right)$ and $f_{n}(x)=\pi_{n}^{2}(x) /\left(x-\xi_{n}^{(n)}\right)$, respectively. Having thus accounted for at least $n+1$, hence exactly $n+1$, nodes $x_{j}^{(n)}$, the interlacing property is established.

THEOREM 2. We have

$$
A_{i}^{(n)}=H_{i}^{(n)}+\frac{h_{n}}{k_{n} Q_{2 n+1}^{\prime}\left(\xi_{i}^{(n)}\right)}, \quad i=1, \ldots, n,
$$

where $H_{i}^{(n)}$ are the Christoffel numbers for the weight function $w(x)$. The inequalities

$$
A_{i}^{(n)}<H_{i}^{(n)}, \quad i=1, \ldots, n,
$$

hold if and only if the nodes $x_{j}^{(n)}$ and $\xi_{i}^{(n)}$ interlace.

Proof. Letting

$$
f_{i}(x)=q_{n+1}(x) \pi_{n}(x) /\left(x-\xi_{i}^{(n)}\right), \quad i=1, \ldots, n,
$$

in (1.1), we have

$$
\int_{a}^{b} w(x) f_{i}(x) d x=A_{i}^{(n)} Q_{2 n+1}^{\prime}\left(\xi_{i}^{(n)}\right)
$$

Applying the $n$-point Gaussian rule to $f_{i}$, and noting that the remainder is

$$
\frac{f_{i}^{(2 n)}(\xi)}{(2 n) ! k_{n}^{2}} \int_{a}^{b} w(x) \pi_{n}^{2}(x) d x=\frac{h_{n}}{k_{n}}
$$

we find that

$$
\int_{a}^{b} w(x) f_{i}(x) d x=H_{i}^{(n)} Q_{2 n+1}^{\prime}\left(\xi_{i}^{(n)}\right)+h_{n} / k_{n} .
$$

From the last two relations, $(2.5)$ follows, since again, $Q_{2 n+1}^{\prime}\left(\xi_{i}^{(n)}\right) \neq 0$.

If the nodes $x_{j}^{(n)}$ and $\xi_{i}^{(n)}$ interlace, then $Q_{2 n+1}^{\prime}\left(\xi_{i}^{(n)}\right)<0$ for all $i$, proving (2.6). Vice versa, if (2.6) holds, consider

$$
f_{j}(x)=q_{n+1}^{2}(x) /\left(\left(x-x_{j+1}^{(n)}\right)\left(x-x_{j}^{(n)}\right)\right), \quad j=1, \ldots, n .
$$


By applying (1.1) we have

$$
\int_{a}^{b} w(x) f_{j}(x) d x=\sum_{i=1}^{n} A_{i}^{(n)} f_{j}\left(\xi_{i}^{(n)}\right)
$$

and from the $n$-point Gaussian rule, with remainder, similarly as above,

$$
\int_{a}^{b} w(x) f_{j}(x) d x=\sum_{i=1}^{n} H_{i}^{(n)} f_{j}\left(\xi_{i}^{(n)}\right)+h_{n} / k_{n}^{2} .
$$

By subtracting (2.9) from (2.10) we obtain

$$
\sum_{i=1}^{n}\left(H_{i}^{(n)}-A_{i}^{(n)}\right) f_{j}\left(\xi_{i}^{(n)}\right)=-h_{n} / k_{n}^{2}<0 .
$$

Since $H_{i}^{(n)}-A_{i}^{(n)}>0, i=1, \ldots, n$, inequality (2.11) is possible only if at least one of the numbers $f_{j}\left(\xi_{i}^{(n)}\right)$ is negative. This means that at least one $\xi_{i}^{(n)}$, say $\xi_{i j}^{(n)}$, satisfies the inequality

$$
x_{j+1}^{(n)}<\xi_{i_{j}}^{(n)}<x_{j}^{(n)}, \quad j=1, \ldots, n,
$$

which, as before, implies the interlacing property.

Clearly, Theorems 1 and 2 both apply to the extended Gaussian quadrature rules, if one chooses $q_{n+1}(x)=p_{n+1}(x)$.

3. Numerical Results. We have constructed the polynomial $p_{n+1}(x)$ satisfying condition (1.2) for $w(x)=\left(1-x^{2}\right)^{\lambda-1 / 2}, \lambda=0(.5) 5,8$, up to $n=20$, by using an algorithm similar to the one described in [4]. When the zeros of these polynomials are all real, the corresponding weights $A_{i}^{(n)}$ and $B_{j}^{(n)}$ were computed by means of $(2.1)$ and (2.5). For all rules thus obtained, the nodes always satisfy the interlacing property; nevertheless, in some cases we find negative weights $A_{i}^{(n)}$. Cases of complex zeros also occur. A brief list of the values of $\lambda$ and $n$, for which negative weights and complex zeros were observed, is reported in the following table (where $k(i) l$ denotes the sequence of integers $k, k+i, k+2 i, \ldots, l)$.

\begin{tabular}{|r|c|c|}
\hline$\lambda$ & $n\left(A_{i}^{(n)}<0\right)$ & $n$ (complex zeros) \\
\hline 4 & 13,15 & \\
4.5 & $7(2) 13,16$ & $15,17,19$ \\
5 & $7,9,14,16$ & $11(2) 19,20$ \\
8 & $3,5,6,8$ & $7,9(1) 20$ \\
\hline
\end{tabular}

Similarly, we examined $w(x)=e^{-x^{2}}$ and $w(x)=e^{-x}$, again up to $n=20$. In the first case, studied already in [7] up to $n=10$, we have confirmed that extended Gaussian rules exist only for $n=1,2,4$. For the second weight function, when $n=1$, the zeros of $p_{2}(x)$ are real, but one is negative, while for $2 \leqslant n \leqslant 20$ some of the zeros are complex.

4. Extended Gauss-Chebyshev Rules. The extension of Gauss-Chebyshev rules can be carried out explicitly by virtue of the identity 


$$
2 T_{n}(x) U_{n-1}(x)=U_{2 n-1}(x),
$$

where $T_{n}(x)$ and $U_{n}(x)$ are the $n$ th-degree Chebyshev polynomials of first and second kind, respectively.

When $w(x)=\left(1-x^{2}\right)^{-1 / 2}$ we may choose $p_{n+1}(x)=2^{-n+1}\left(x^{2}-1\right) U_{n-1}(x)$, $n \geqslant 2$, and (1.1) becomes the Gauss-Chebyshev rule of closed type (see for example [1])

$$
\int_{-1}^{1}\left(1-x^{2}\right)^{-1 / 2} f(x) d x=\frac{\pi}{2 n}\left[\sum_{i=1}^{2 n-1} f\left(x_{i}^{(n)}\right)+\frac{1}{2} f(-1)+\frac{1}{2} f(1)\right]+R_{n}(f),
$$

$$
n \geqslant 2 \text {, }
$$

where

$$
x_{i}^{(n)}=\cos \frac{i \pi}{2 n}, \quad i=1,2, \ldots, 2 n-1 .
$$

$p_{n+1}(x)$ satisfies the required orthogonality condition (1.2) by virtue of (4.1). As a matter of fact, (1.2) holds for all $k \leqslant 2 n-2, n \geqslant 2$. Since the coefficients $A_{i}^{(n)}$, $B_{j}^{(n)}$ are uniquely determined, they must be as in (4.2), which is known to have not only degree $3 n+1$, but in fact degree $4 n-1$. For $n=1$ we have $p_{2}(x)=x^{2}-3 / 4$ and (1.1) coincides with the 3-point Gauss-Chebyshev rule.

A natural way of iterating the process is to add $2 n$ new nodes, namely the zeros of $T_{2 n}(x)$, so that, by virtue of (4.1), the new rule will have as nodes the zeros of $\left(x^{2}-1\right) U_{4 n-1}(x)$ and polynomial degree $8 n-1$. In general, after $p$ extensions, having reached a rule with $2^{p} n+1$ nodes, we add $2^{p} n$ new nodes, namely the zeros of $T_{2 p_{n}}(x)$, to get a rule of the type (4.2) with $2^{p+1} n+1$ nodes and polynomial degree $2^{p+2} n-1$.

In a similar way we may extend the Gaussian quadrature rule for the weight function $w(x)=\left(1-x^{2}\right)^{1 / 2}$. Recalling again (4.1), we choose $p_{n+1}(x)=2^{-n} T_{n+1}(x)$, and obtain

$$
\int_{-1}^{1}\left(1-x^{2}\right)^{1 / 2} f(x) d x=\frac{\pi}{2(n+1)} \sum_{i=1}^{2 n+1}\left(1-\left[x_{i}^{(n)}\right]^{2}\right) f\left(x_{i}^{(n)}\right)+R_{n}(f),
$$

the Gaussian rule constructed over the $2 n+1$ zeros

$$
x_{i}^{(n)}=\cos \frac{i \pi}{2(n+1)}, \quad i=1,2, \ldots, 2 n+1,
$$

of the polynomial $U_{2 n+1}(x)$. It has polynomial degree $4 n+1$. As before, the process may be iterated.

Acknowledgement. I am grateful to Professor Walter Gautschi for many constructive suggestions and constant guidance during this work.

Department of Computer Sciences

Purdue University

West Lafayette, Indiana 47907

1. M. M. CHAWLA, "Error bounds for the Gauss-Chebyshev quadrature formula of the closed type," Math. Comp., v. 22, 1968, pp. 889-891. MR 39 \#1113.

2. P. J. DAVIS \& P. RABINOWITZ, Methods of Numerical Integration, Academic Press, New York, 1975. 
3. A. S. KRONROD, Nodes and Weights for Quadrature Formulae. Sixteen-Place Tables, "Nauka", Moscow, 1964; English transl., Consultants Bureau, New York, 1965. MR 32 \#597, \#598.

4. T. N. L. PATTERSON, "The optimum addition of points to quadrature formulae," Math. Comp., v. 22, 1968, pp. 847-856; Addendum, ibid., v. 22, 1968, no. 104, loose microfiche suppl. C1-C11. MR $39 \# 3701$.

5. T. N. L. PATTERSON, "Algorithm 468-Algorithm for automatic numerical integration over a finite interval," Comm. $A C M$, v. 16, 1973, pp. 694-699.

6. R. PIESSENS, "An algorithm for automatic integration," Angewandte Informatik, v. 9, 1973, pp. 399-401.

7. Ju. S. RAMSKIII, "The improvement of a certain quadrature formula of Gauss type," Vycisl. Prikl. Mat. (Kiev), Vyp. 22, 1974, pp. 143-146. (Russian) MR 50 \#6121.

8. W. SQUIRE, Integration for Engineers and Scientists, American Elsevier, New York, 1970.

9. G. SZEGÖ, "Über gewisse orthogonale Polynome, die zu einer oszillierenden Belegungsfunktion gehören," Math. Ann., v. 110, 1934, pp. 501-513. 